\title{
Psychometric properties of the Community Mental Health Team effectiveness questionnaire (CMHTEQ)
}

\author{
ANNE REES ${ }^{1}$, CHRIS STRIDE $^{2}$, DAVID A SHAPIRO ${ }^{1}$, ANN RICHARDS $^{1} \&$ CAROL \\ BORRILL ${ }^{3}$
}

${ }^{1}$ Psychological Therapies Research Centre, University of Leeds, ${ }^{2}$ Institute of Work Psychology, University of Sheffield \& ${ }^{3}$ Aston Business School, University of Aston

\begin{abstract}
The Community Mental Health Team effectiveness questionnaire (CMHTEQ) is a 27-item measure of Community Mental Health Team (CMHT) effectiveness for completion by team members. It was constructed following a stakeholder conference representing the following constituencies: clinicians, users and carers, mental health researchers, policy makers and managers. We present a psychometric analysis of the CMHTEQ, based on the responses of 1450 (response rate: 75\%) CMHT staff of a sample of 113 CMHTs recruited from all Trusts providing community mental health care across four English NHS Regions. Three factors emerged from an exploratory analysis of $50 \%$ of the data, upheld by a confirmatory analysis of the remaining data: meeting external requirements; internal team processes; evidence and feedback. Factor scales exhibited acceptable internal reliabilities. The CMHTEQ meets the need for a measure of the effectiveness of CMHTs, as perceived by their members, for use in research studies of the environment and effectiveness of mental health care, and by service managers or CMHTs seeking to monitor or track performance change over time.
\end{abstract}

\section{Introduction}

Community mental health teams (CMHTs) face many challenges. They are tasked with complex statutory and professional responsibilities (Peck \& Parker, 1998). The demands of a primary care-led NHS often conflict with the policy imperatives of the sensitive area of risk management relating to severe mental health problems (Onyett et al., 1995). In addition, the voice of service users gains strength, adding to workload and pressures. Team members are employed within two very different bureaucracies, those of health and social care, and come from diverse professional backgrounds. However, the development of joint commissioning approaches between health and local authority social services requires them to function as integrated teams (Hannigan, 1999). Their constituent professions may jibe at the adjustments this requires (Mistral \& Velleman, 1997), for which their training may not prepare them well.

The current policy agenda is increasingly outcomes-focused. Accordingly, CMHTs

Address for Correspondence: Anne Rees, Psychological Therapies Research Centre, University of Leeds, 17 Blenheim Terrace, Leeds LS2 9JT, United Kingdom. Tel: 0113233 1955; Fax: 0113233 1956; E-mail: anner@psychology.leeds.ac.uk. 
are required to monitor their performance (Bhugra et al., 1995) and effectiveness, as a strong commitment to monitoring and evaluation is considered essential for adequate management of CMHT services (Carter et al., 1997). The competition for resources amongst elements of health and social care provision requires that each provide data to demonstrate the value of its contribution. More positively, effectiveness measures may also bring some clarity to teams' efforts to chart their own progress towards meeting diverse expectations.

The organisation of CMHTs is central to theirfunctioning(Bhugra et al., 1995; Onyett, 1997). Their core rationale is to bring together a range of professions in order to deliver more effective care co-ordination than could be achieved without an integrated, multidisciplinary team. Achieving that integration is by definition an organisational task (Onyett, 1995; Pincus et al., 1996), requiring that the team be more than the sum of its diverse constituent members acting individually.

User and carer perspectives are increasingly important. The National Health Service Patients' Charter for Mental Health Services (Department of Health, 1997) sets out rights and expected standards of service for users and potential users of these services. It aims to ensure that the NHS 'listens and acts upon people's views and needs'. A continuing pushfor users and carers to be involved in decisions relating to mental health care (Faulkner, 1997), and also, to be included at the level of planning and developing services, presents a further challenge to teams which deliver integrated care within the Care Programme Approach(Department of Health, 1995; 1990).

There are at least two important reasons for developing models and measures of community mental health team effectiveness. First, it is essential to monitor different or changing models of care, to define which accountability structure is in place for a given team (Ovretveit, 1993), and to enable the implementation of evidence-based practice (Bhugra et al., 1995). Second, a sense of effective activity is known to be associated with higher levels of subjective well-being (Poulton \& West, 1994), a very important consideration for practitioners in CMHTs at the sharp end of health care delivery (Harper \& Minghella, 1997; Prosser et al., 1996). Yet it is a difficult task to define appropriate parameters with which to describe such a complex and challenging environment.

In view of all the competing pressures, how might the effectiveness of CMHTs be most appropriately gauged? The diverse demands on CMHTs may be best represented by the constituency approach (Connally et al., 1980), which acknowledges and indeed capitalises on the differences among stakeholders. Such an approach has previously been applied to Primary Health Care Team environments (Poulton \& West, 1994). Effectiveness of CMHTs can only be satisfactorily assessed using criteria designed to reflect the full range of demands and expectations teams are required to meet. Thus it would be mistaken, for example, to define effectiveness solely in terms of attaining a given level of clinical outcomes. This criterion fails to capture much of what the diverse stakeholders in CMHTs would consider relevant to evaluating the effectiveness of a team. For example, how effectively does a team ensure that it meets the needs of users and carers? How well does the team monitor the success of its work? Is the team effectively organised? No tool exists to measure effectiveness in CMHTs from the perspectives of their multiple stakeholders.

We recently reported on a stakeholder workshop following the constituency approach 
that generated 27 effectiveness criteria representing the views, experiences and opinions of clinicians, users and carers, mental health researchers, policy makers and managers (Richards \& Rees, 1998). The Community Mental Health Team Effectiveness Questionnaire (CMHTEQ) comprises a single item to tap each of these 27 criteria. We now present the psychometric properties of the measure, including factor structure and scale reliability, using data from a large sample of members of a representative set of CMHTs.

\section{Method}

\section{Construction of the CMHTEQ}

Effectiveness criteria were generated using an iterative process within the constituency model approach (Connally et al., 1980). We describe elsewhere the implementation of this in the construction of the CMHTEQ (Richards \& Rees, 1998); 27 criteriafor evaluating CMHT effectiveness were defined. Within the CMHTEQ, each criterion is presented in the form of a statement, neither positively nor negatively framed. Each statement is clarified by additional concrete examples of elements of practice which individuals may use to aid their rating. A 5-point Likert-type scale is used to rate team members' perceptions of how effective the team is on each criterion. For example, the first item of the CMHTEQ is reproduced in Figure 1.

\section{Gaining access to CMHTs}

Initially, chief executives of 101 community mental health Trusts in four regions, Northern and Yorkshire, North West, Trent, and North Thames, were approached, to inform them of the study and to encourage participation of all CMHTs managed by that Trust. The aim was to limit the geographical spread for logistic reasons, while also drawing CMHTs from a representative range of socioeconomic locations, skill mix and client base. Three months after the first mailshot, follow-up letters were sent to all Trusts not responding. Of the 101 approached, 81 responded: 11 had no community adult mental health services; 12 declined to participate and the remaining 58 provided names and contacts for all CMHTs managed. The main reasons at Trust level for not participating were either that (a) the Trust was in the process of reorganisation (seven Trusts); (b) caseloads were such that teams were too busy (three Trusts); or (c) the teams were already taking part in other research (two Trusts).

With the CMHT contacts provided, we made direct contact with 162 CMHTs, inviting participation in the study after consensus to participate had been achieved within each team. This procedure made the access process longer, while team contacts took the issue of participation to their team meeting. Nevertheless, a small proportion of CMHTs were

Accessibility of the service to users and carers has been identified as a measure of CMHT effectiveness.

(For example: identification and contactability of a key worker; clear referral procedures; time taken to respond to users and or carers; a clear point of access.)

Not at all

Overall, to what extent does your CMHT make services accessible to users and carers?
To a great extent

$\begin{array}{lllll}1 & 2 & 3 & 4 & 5\end{array}$

Figure 1: Layout of a sample item from the CMHT effectiveness questionnaire 
directed to participate by Trust management. The final number of participating teams was 113 from 45 trusts. Details of the sample are shown in Table 1. At different stages of the access procedure, it was open to Trusts or CMHTs to refuse to participate; the sample was therefore made up of volunteering CMHTs. We performed a post hoc check on socioeconomic representativeness, which indicated that the whole range of deprivation scores was represented(Mental Illness Needs Index (MINI; Glover et al., 1998) range 91.3 (low need) to 118.5 (high need), mean 103.3).

Table 1: Sample profile

\begin{tabular}{|l|c|c|}
\hline & $\begin{array}{c}\text { Number } \\
\text { of teams }\end{array}$ & \% \\
\hline Region & 32 & 28 \\
Northern \& Yorkshire & 32 & 28 \\
North West & 23 & 20 \\
Trent & 26 & 23 \\
North Thames & 22 & 19 \\
Team size (members) & 35 & 31 \\
5-10 & 27 & 24 \\
$11-15$ & 21 & 19 \\
$16-20$ & 8 & 7 \\
$21-30$ & & \\
$30+$ & 19 & 17 \\
Deprivation score (MINI) & 28 & 25 \\
No data & 34 & 30 \\
Low need (<100) & 32 & 28 \\
Medium (100-105) & \\
High need (>105) &
\end{tabular}

\section{Distribution and return of questionnaires}

The named contact for each of the 113 participating CMHTs provided a comprehensive list of all team members, which included all personnel attending regular team meetings. CMHTs with a response rate lower than $50 \%$ were contacted monthly to try to increase the rate of response, which resulted in only 6 of the 113 achieving afinal response rate of under $50 \%$. Survey questionnaires were sent to 1925 named inviduals, with returns from $1450(75 \%)$. The return rates for professional groups were: administrative staff $57 \%$; community psychiatric nurses $82 \%$; occupational therapists $83 \%$; psychiatrists $55 \%$; clinical psychologists $90 \%$; social workers $53 \%$; and support workers $68 \%$. Overall, 925 women $(64 \%)$ were included in the sample, and the mean age was 40 (SD 8.37).

\section{Analysis}

Factor analysis was used to generate and evaluate the bestfitting model to describe the associations among the 27 effectiveness variables as these were rated by the 1446 respondents in our sample. The sample was randomly split into two halves. A model for the data was developed using exploratory factor analysis (EFA) of the responses of the first subsample. This model was then validated via confirmatory factor analysis (CFA) of the responses of the second subsample. The Principal Components method of factor extraction was used in the EFA.

Any case with missing data on any variable was deleted, causing a loss of $13 \%$ from an original sample size of 1446. Factors were extracted until they accounted for less than the variance of a single, standardised variable (i.e. eigen values greater than 1). Following standard factor-analytic procedure, the factors were rotated to seek the best fit. This maximises the high loadings of variables on factors and minimises the low ones. Both orthogonal and oblique rotations were examined. Orthogonal rotations assume that the factors are uncorrelated, whilst oblique rotations seek the most parsimonious account of the data whilst allowing the factors to be intercorrelated. To evaluate oblique rotations, we used the pattern matrix, which depicts the relationship of the observed variables to the factors taking account of intercorrelations between the factors. 
The CFA of the second subsample served to test the fit of the model and to evaluate statistically the extent to which allowing the factors to be correlated is justifiable (Dunn et al., 1993). This was carried out using the AMOS software package (Arbuckle, 1996). The baseline model was a null model with zero covariances between all 27 items (Bentler \& Bonett, 1980).

The fit of each model to the data was initially assessed by the chi-squared statistic and its ratio to the degrees of freedom, with a non-significant model chi-squared statistic indicating a good fit of the model to the data. However, since large sample sizes can result in highly significant chi-squared statistics even when the model's departure from the data is very slight, the Comparative Fit Index (CFI) and the Non-Normed Fit Index (NNFI) were used to evaluate the fit of the specified model. Both take values between 0 and 1 , with a value of 1 indicating a perfectly fitting model. Bentler (1992) suggested that a value of greater than 0.90 is required to indicate an adequate fit to the data.

Reliability analyses were carried out on the three groups of items to determine their suitability for use as scales.

\section{Results}

\section{Exploratory factor analysis}

Three factors with eigen values greater than 1 were extracted by the Principal Components method. They accounted for $44.3 \%$, $4.6 \%$, and $4.0 \%$ of the variance, respectively. Oblique rotation produced a more clearly interpretable solution than orthogonal rotation. This is shown in Table 2. It revealed high correlations (all greater than 0.45) among the three factors, as shown in Table 3 . Factor 2 was negatively correlated with the other two factors, which were positively correlated. Interpretation of the factors was not attempted prior to their confirmation with the second subsample.

\section{Confirmatory factor analysis}

Three-factor models with and without correlations between the factors were tested. Both models were based on the largest loading recorded for each variable in Table 2, specifying variables $1,10,11,12,13,15,20$, $21,23,24,26$, and 27 as loading on factor 1; variables $2,3,5,6,7,14,16,17,22$ and 25 loading on factor 2; and variables 4, 8, 9, 18 and 19 loading on factor 3. Model 1 allowed correlations between the factors. Model 2 allowed no such correlations, hypothesising that the factors are independent of each other.

As expected given the large sample size, both models yielded significant chi-square statistics: 1088.9 with 321 df for Model 1; and 2392.3 with 324 df for Model 2. The statistically significant reduction of 1303 , $\mathrm{df}=3, p<0.0001$, in the chi-squared statistic, indicates that Model 1's allowance of intercorrelation among the factors was statistically justifiable. Consistent with this, the fit indices showed that Model 1, with a CFI of 0.92, and an NNFI $=0.91$, was an acceptable fit to the data, while model 2, with a CFI of 0.77 and an NNFI of 0.75 , was not.

The best available model was therefore Model 1, presented in Table 4. To aid understanding, the variables are grouped by their respective factors, and ordered within each factor from those with the highest to the lowest loading on that factor.

Table 5 shows that the factors were even more highly correlated than in the exploratory analysis (Table 3). Despite this, however, clearly interpretable differences were found in terms of content between the factors. Factor 1 refers mainly to meeting external requirements. Factor 2 refers mainly to internal team processes. Factor 3 is best captured as evidence andfeedback. 
Table 2: Exploratory factor analysis: Pattern matrix

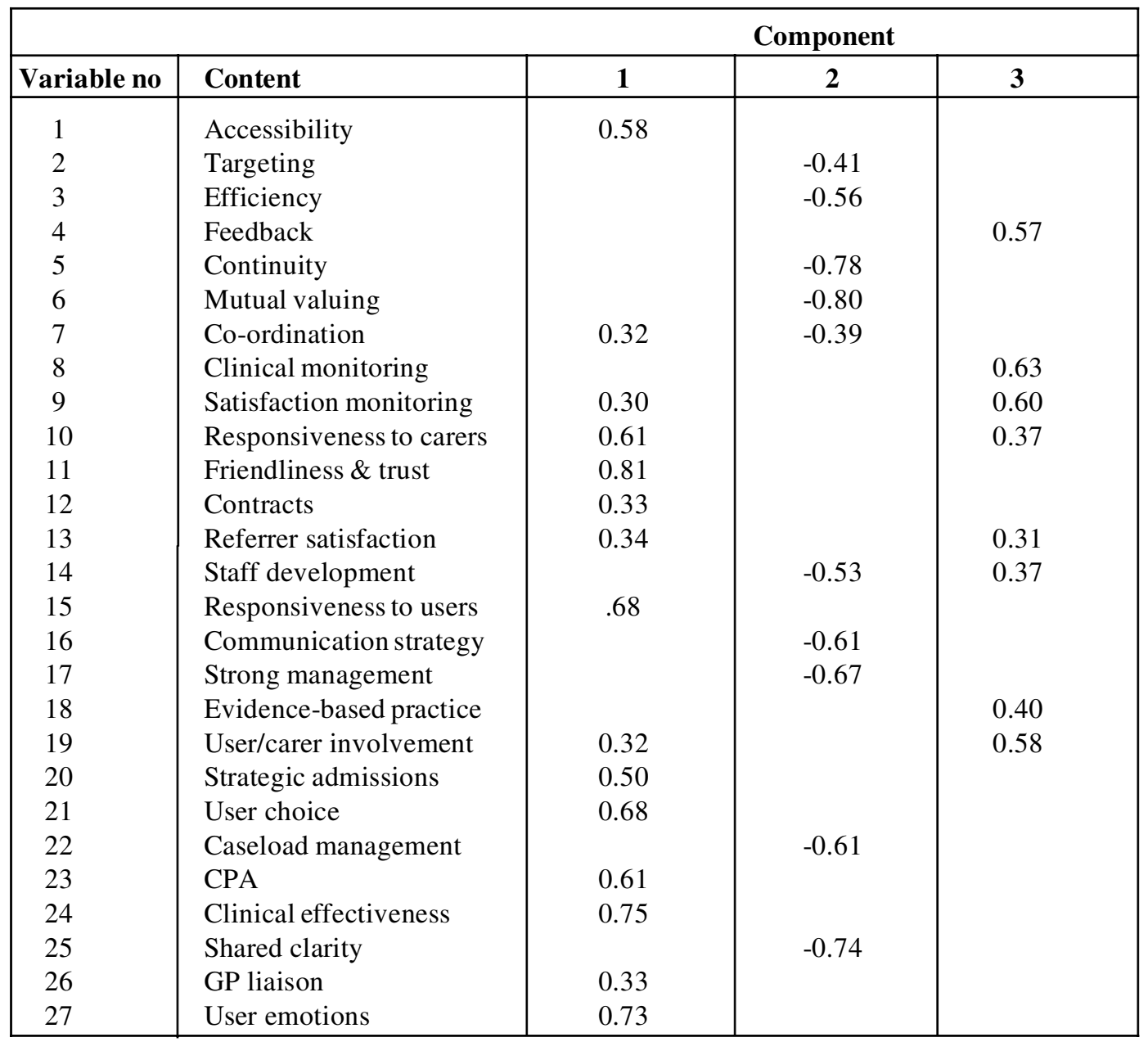

Note: Extraction by principal component analysis, rotation using Oblimin with Kaiser normalisation; rotation converged in 20 iterations.

Table 3: Exploratory factor analysis:

Correlations between factors

\begin{tabular}{|l|l|l|l|}
\hline Factor & $\mathbf{1}$ & $\mathbf{2}$ & $\mathbf{3}$ \\
\hline 1 & & -0.66 & 0.51 \\
\hline 2 & & & -0.47 \\
\hline
\end{tabular}

\section{Supplementary analyses}

Additional sets of analyses were carried out to check the robustness of the findings. Because of the substantial loss of respondents $(13 \%)$ resulting from listwise deletion of data when performing EFA on the construction sample, we performed the same analysis with pairwise deletion. As with the listwise deleted sample, three factors were extracted, accounting for $53 \%$ of the variance; moreover following an oblique rotation, the pattern matrix indicated a very similar model. However, small changes occurred in the loadings of three variables which had weak loadings on all three factors, with variable 12 now loading most strongly (-0.31) onto factor 2 , variable 13 now loading most strongly $(0.35)$ onto factor 3 , and variable 26 now loading 
Table 4: Confirmatory factor analysis: Pattern matrix

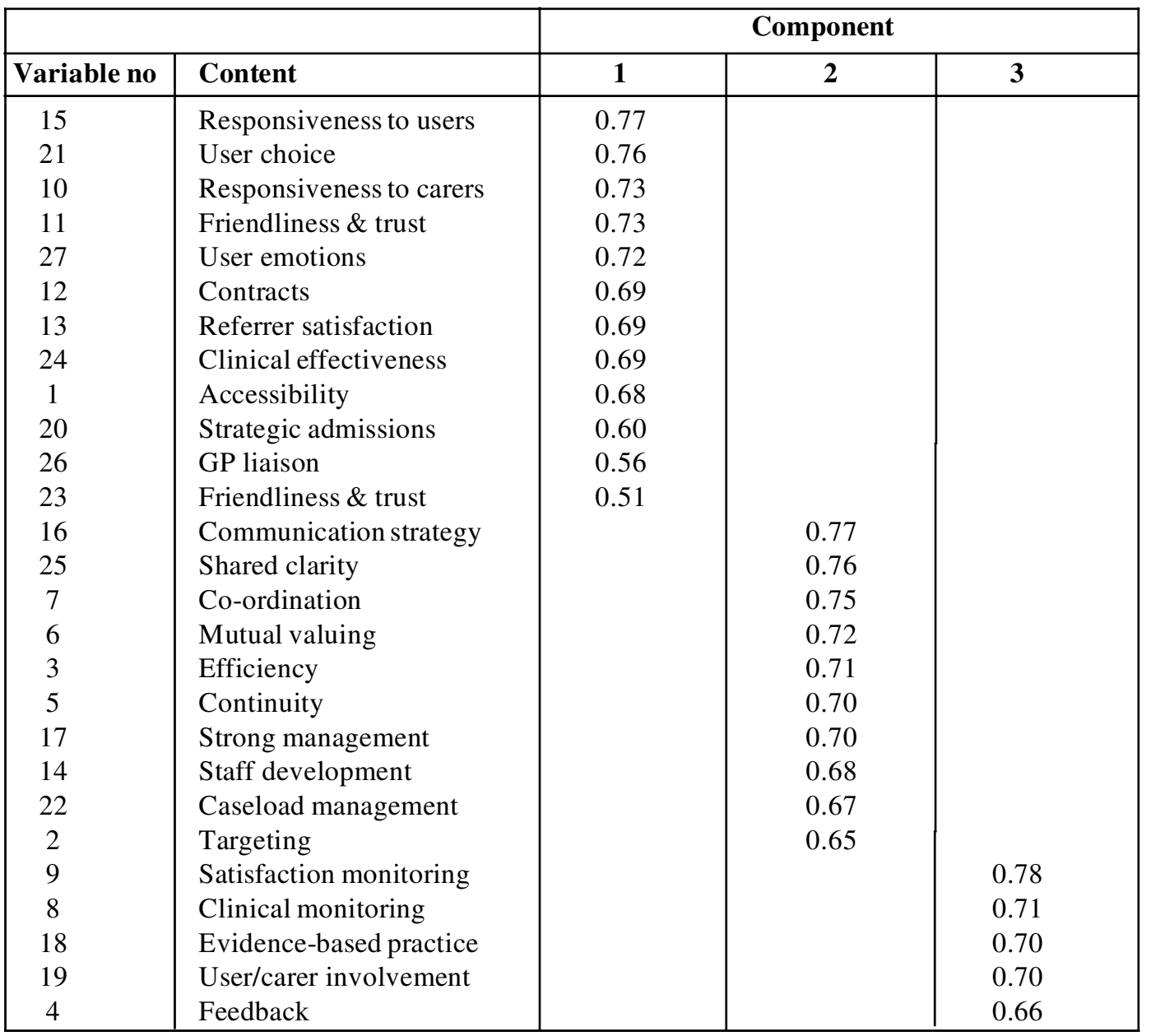

Table 5: Confirmatory factor analysis: Correlations between factors

\begin{tabular}{|c|c|c|c|}
\hline Factor & $\mathbf{1}$ & $\mathbf{2}$ & $\mathbf{3}$ \\
\hline 1 & & 0.89 & 0.91 \\
\hline 2 & & & 0.85 \\
\hline
\end{tabular}

most strongly $(-0.32)$ onto factor 2 . Confirmatory analyses were run on our validation sample for all these possible variations, with fit indices and chi-squared statistics listed in Table 6. Since the models are not nested within the initial model we cannot test them directly against it, but none of them produced higher fit indices than our initial model.
In addition, in light of the very high factor intercorrelations, we also fitted a single factor model, and a 2-factor model in which factors 1 and 3 were combined. Neither of these fitted the data as well as the 3-factor model. The one factor model had a CFI of 0.88 and NNFI of 0.87 , the 2 -factor model had a CFI of 0.91 and an NNFI of 0.90.

\section{Reliability analysis}

Considering each of the three sets of items as a scale, internal consistency of all scales was high. Cronbach's alpha coefficients were 0.91 for meeting external requirements, 
Table 6: Supplementary analyses

\begin{tabular}{|l|l|l|l|}
\hline Change from Model 1 above & Chi-squared statistic & CFI & NNFI \\
\hline Variable 12 loads on to factor 2 & 1125.72 on 321df & 0.911 & 0.902 \\
Variable 13 loads on to factor 3 & 1094.96 on 321df & 0.914 & 0.906 \\
Variable 26 loads on to factor 2 & 1118.40 on 321df & 0.911 & 0.903 \\
\hline
\end{tabular}

0.91 for internal team processes, and 0.83 for evidence and feedback.

\section{Discussion}

Rather than adopting a theoretical orientation to enable the identification of components of effective delivery of CMHT care, the internalised knowledge and experience of all stakeholders in community mental health care was gathered together to generate the criteria to be included within the CMHTEQ. This captured the depth and complexity of this particular health care environment, especially as concrete, measurable indices were provided alongside the stated criteria of effectiveness. Thus, the systematic process of the stakeholder conference assures the instrument of a high level of face validity.

The psychometric analysis presented here is based on a large sample $(N=1450)$ and acceptable response rate $(75 \%)$. The three factors emerging were robust, as revealed by CFA and by the reliability of their respective scales. Their contents capture three salient dimensions of CMHT effectiveness as specified by stakeholders: meeting external requirements; internal team processes; and evidence and feedback.

The first factor, meeting external requirements, reflects the importance of CMHTs being seen to fulfil the demanding role with which they are charged by both health and social services, with its high-profile impact on the community. The second factor, internal team processes, reflects the considerable challenges of multiprofessional and multiagency integration within the team, and the interdependence of the diversely-trained team members in achieving their common purposes. The final factor, evidence and feedback, aptly captures current demand for evidence-based practice, and for services to be open to learnings from experience and feedback.

The CMHTEQ's ability to measure these three factors will be valuable to research studies of the environment and effectiveness of mental health care. This might consider, for example, what needs to be done to enhance each of the three dimensions of CMHT effectiveness. The instrument may also be useful to service managers or CMHTs themselves, seeking to monitor or track performance change over time, via repeated, systematic self-assessment by team members of their team's effectiveness. Trusts providing community mental health services could use the CMHTEQ to evaluate planned organisational changes, whether to the composition, internal working procedures, or interface with other elements of health and social services, pertaining to their CMHTs.

This study had some limitations. We lost $30 \%$ of CMHTs from the potential sample, usually because the CMHT could not achieve consensus or did not want to participate in further stages of the research. This meant that the teams who did participate were selfselecting, although post-hoc checks revealed that socioeconomic representativeness was good. The response rate was goodfor a postal questionnaire methodology, though still not perfect. While social work professionals 
were well represented at the stakeholder workshop and played a full part in the consensus process, it was felt by some social workers responding that the questionnaire reflected a bias towards a specifically health environment.

The high intercorrelations among the factors might be thought to reduce the utility of distinguishing them. However, the fit statistics indicated the superiority of the 3 -factor over 2- and 1-factor solutions. This suggests that, whilst it would be possible to represent the data as simply differentiating 'good' from 'poor' teams overall, acknowledging the existence of three dimensions produces a better account of the data and thus carries real descriptive power.

Several CMHTs participating in the effectiveness survey are now using the CMHTEQ to track their performance independently. The authors would like to encourage teams, individuals or managers to contact them with a view to collaboration in implementing the CMHTEQ in their specific environment.

\section{Acknowledgement}

This study was supported by a grant from the UK Department of Health's Policy Research Branch, Human Resources and Effectiveness Programme, to Michael West, Simon Garrod and David A Shapiro

\section{References}

Arbuckle, J.L. (1996). AMOS users guide version 3.6. SPSS.

Bentler,P.M. (1992). On the fit of models to covariances and methodology, to the Bulletin. Psychological Bulletin, 112, 400-404.

Bentler,P.M. \& Bonett, D.G. (1980). Significancetests and goodness of fit in the analysis of covariance structures. Psychological Bulletin, 88, 588-606.

Bhugra, D., Bridges, K. \& Thompson, C. (1995). Caring for a community: The community care policy of the Royal College of Psychiatrists. London: Royal College of Psychiatrists.
Carter, M.F., Evans, K.E., Crosby, C., Prendeergast, L.A. \& De Sousa Butterworth, K.A. (1997). The all-Wales community mental health team survey. Bangor: Health Services Research Unit.

Connally, T., Conlan, E.J. \& Deutsch, S.J. (1980). Organisational effectiveness: a multiple constituency approach. Academy of Management Review, 5, 211-217.

Department of Health (1997). The Patient's Charter: mental health services. London: Department of Health.

Department of Health(1995).Building bridges: a guide to arrangementsfor inter-agency working for the care and protection of severelymentally ill people. London: HMSO.

Department of Health (1990). The Care Programme Approachfor people with a mental illness referred to the specialist psychitric services. $\mathrm{HC}(90) 23 /$ LASSL(90)11, Lonson: Department of Health.

Dunn, G., Everitt, B. \& Pickles, A. (1993). Modelling covariances and latent variables using EQS. London: Chapman and Hall.

Faulkner,A. (1997). Knowing ourown minds: A survey of how people in emotional distress take control of their lives. London: Mental Health Foundation.

Glover, G.R., Robin, E., Emami, J. \& Arabscheibani, G.R. (1998). A needs index for mental health care. Social Psychiatry and Psychiatric Epidemiology, 33, 89-96.

Hannigan, B. (1999). Joint working in community mental health: prospects and challenges. Health and Social Care in the Community, 7, 25-31.

Harper, H. \& Minghella, E. (1997). Pressures and rewards of working in community mental health teams. Mental Health Care, 1, 18-21.

Mistral, W. \& Velleman, R. (1997). Community mental health teams: the professionals' choice? Journal of Mental Health, 6, 125-140.

Onyett, S. (1997). The challenge of managing community mental healthteams. Health and Social Care in the Community, 5, 40-47.

Onyett, S. (1995). Responsibility and accountabilityin community mental health teams. Psychiatric Bulletin, 19, 281-285.

Onyett, S., Pillinger, T. \& Muijen, M. (1995). Making community mental health teams work. London: Sainsbury Centre for Mental Health.

Ovretveit, J. (1993). Co-ordinating community care: multi-disciplinary teams and care management. Buckingham: Open University Press.

Peck, E. \& Parker,E. (1998). Mental health in the NHS: Policy and practice 1979-98. Journal of Mental Health, 7, 241-259. 
Pincus, H.A., Zarin, D.A. \& West, J.C. (1996). Peering into the 'black box': measuring outcomes of managed care. Archives of General Psychiatry, 53, 870-877.

Poulton, B. \& West, M.A. (1994). Primary health care team effectiveness: developing a constituency approach. Health \& Social Care, 2, 77-84.
Prosser, D, Johnson, S., Kuipers, E., Szmukler, G., Bebbington, P. \& Thornicroft, G. (1996). Mental health, 'burnout' and job satisfactionamong hospital and community-basedmental health staff. British Journal of Psychiatry, 169, 334-337.

Richards, A. \& Rees, A. (1998). Developing criteria to measure the effectiveness of community mental health teams. Mental Health Care, 2, 14-17. 